\title{
11-COLORED KNOT DIAGRAM WITH FIVE COLORS
}

\author{
TAKUJI NAKAMURA, YASUTAKA NAKANISHI, AND SHIN SATOH
}

\begin{abstract}
We prove that any 11-colorable knot is presented by an 11-colored diagram where exactly five colors of eleven are assigned to the arcs. The number five is the minimum for all non-trivially 11-colored diagrams of the knot. We also prove a similar result for any 11-colorable ribbon 2-knot.
\end{abstract}

\section{INTRODUCTION}

The $n$-colorability introduced by Fox $[3]$ is one of the elementary notion in knot theory, and its properties have been studied in many papers. In 1999, Harary and Kauffman [5] defined a kind of minimal invariant, $\mathrm{C}_{n}(K)$, of an $n$-colorable knot $K$. It is essential to consider the case that $n$ is an odd prime; in fact, for composite $n$, it is reduced to the cases of odd prime factors of $n$. In this case, we can define a modified version by restricting "effective" $n$-colorings (cf. 6, 12]).

Let $p$ be an odd prime. A non-trivial $p$-coloring $C$ of a knot diagram $D$ is regarded as a non-constant map

$$
C:\{\operatorname{arcs} \text { of } D\} \rightarrow \mathbb{Z} / p \mathbb{Z}=\{0,1, \ldots, p-1\}
$$

with a certain condition. For a $p$-colorable knot $K$, the number $\mathrm{C}_{p}(K)$ is defined to be the minimum number of $\# \operatorname{Im}(C)$ for all non-trivially $p$-colored diagrams $(D, C)$ of $K$. This number has been studied in some papers [2, 4, 7, 8, 10, 11, 13, 15, 17,. In particular, it is shown in [1] that

$$
\mathrm{C}_{p}(K) \geq\left\lfloor\log _{2} p\right\rfloor+2
$$

for any $p$-colorable knot $K$, and the equality holds for $p=3,5,7$ [13, 17 .

For $p=11$, we have $\mathrm{C}_{11}(K) \geq 5$ by the above inequality or [10, Theorem 2.4]. On the other hand, it is proved in 2 that $\mathrm{C}_{11}(K) \leq 6$. If an 11-colored diagram $(D, C)$ satisfies $\# \operatorname{Im}(C)=5$, then there are two possibilities

$$
\operatorname{Im}(C)=\{1,4,6,7,8\},\{0,4,6,7,8\}
$$

up to isomorphisms induced by affine maps of $\mathbb{Z} / 11 \mathbb{Z}$. This split phenomenon is quite different from the cases $p=3,5,7$.

Theorem 1.1. Any 11-colorable knot $K$ satisfies the following.

(i) There is an 11-colored diagram $\left(D_{1}, C_{1}\right)$ of $K$ with $\operatorname{Im}\left(C_{1}\right)=\{1,4,6,7,8\}$.

(ii) There is an 11-colored diagram $\left(D_{2}, C_{2}\right)$ of $K$ with $\operatorname{Im}\left(C_{2}\right)=\{0,4,6,7,8\}$.

We remark that these two sets are common 11-minimal sufficient sets of colors but not universal ones in the sense of [4. By Theorem 1.1. we have the following immediately.

The third author is partially supported by JPSP KAKENHI Grant Number 25400090.

2010 Mathematics Subject Classification. Primary 57M25; Secondary 57Q45.

Key words and phrases. knot, diagram, 11-coloring, virtual arc presentation, ribbon 2-knot. 
Corollary 1.2. Any 11-colorable knot $K$ satisfies $\mathrm{C}_{11}(K)=5$.

This paper is organized as follows. In Section 2, we review the palette graph associated with a subset of $\mathbb{Z} / p \mathbb{Z}$ and its fundamental properties. In Section 3 . we prove Theorem 1.1(i). The starting point of the proof is a modified version of the theorem in 22: For any 11-colorable knot $K$, there is an 11-colored diagram $(D, C)$ of $K$ with $\operatorname{Im}(C)=\{0,1,4,6,7,8\}$. By applying Reidemeister moves to $(D, C)$ suitably, we remove the color 0 from the diagram. Sections 46 are devoted to proving Theorem 1.1(ii). We first remove the color 1 from $(D, C)$ as above by allowing the birth of new colors 3 and 10 in Section 4 and then remove the colors 10 and 3 in Sections 5 and 6 , respectively. In the last section, we prove a similar result for an 11-colorable ribbon 2-knot.

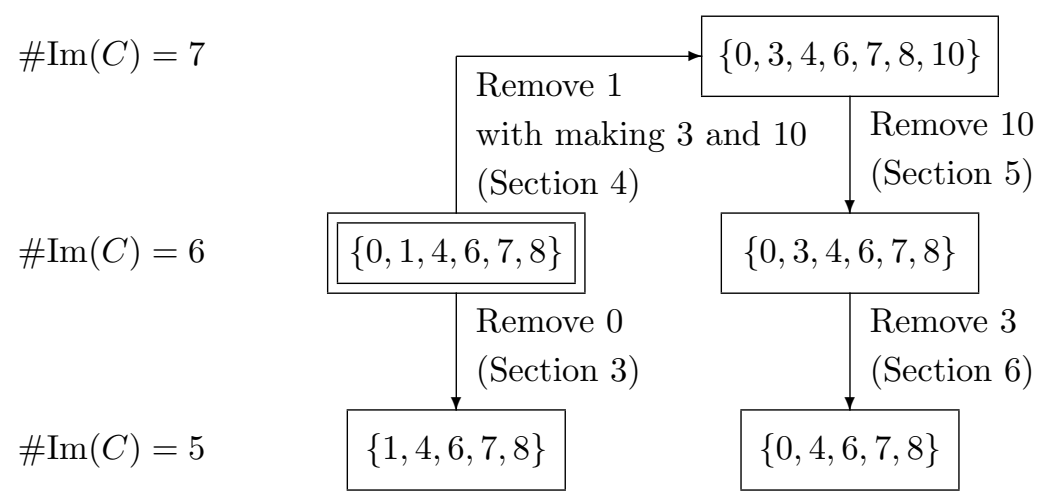

\section{Preliminaries}

Throughout this section, $p$ denotes an odd prime.

Definition 2.1. Let $S$ be a subset of $\mathbb{Z} / p \mathbb{Z}$. The palette graph $G(S)$ of $S$ is a simple graph such that

(i) the vertex set of $G(S)$ is $S$, and

(ii) two vertices $a$ and $b \in S$ are connected by an edge if and only if $\frac{a+b}{2} \in S$.

By assigning $\frac{a+b}{2}$ to every edge joining $a$ and $b$, we regard $G(S)$ as a labeled graph. Such an edge is denoted by $\left\{a\left|\frac{a+b}{2}\right| b\right\}$.

Definition 2.2. For two subsets $S$ and $S^{\prime} \subset \mathbb{Z} / p \mathbb{Z}$, the palette graphs $G(S)$ and $G\left(S^{\prime}\right)$ are said to be isomorphic if there is a bijection $f: S \rightarrow S^{\prime}$ such that $\frac{a+b}{2} \in S$ if and only if $\frac{f(a)+f(b)}{2} \in S^{\prime}$. We denote it by $G(S) \cong G\left(S^{\prime}\right)$.

Lemma 2.3. If $S \subset S^{\prime} \subset \mathbb{Z} / p \mathbb{Z}$, then $G(S)$ is a subgraph of $G\left(S^{\prime}\right)$, which is obtained from $G\left(S^{\prime}\right)$ by deleting the vertices in $S^{\prime} \backslash S$ and the edges whose labels belong to $S^{\prime} \backslash S$.

Proof. This follows from definition immediately.

Theorem 2.4 ([11]). If the palette graph $G(S)$ is connected with $\# S>1$, then we have $\# S \geq\left\lfloor\log _{2} p\right\rfloor+2$. 
Lemma 2.5. Let $S$ be a subset of $\mathbb{Z} / p \mathbb{Z}$ such that $G(S)$ is connected with $\# S=$ $\left\lfloor\log _{2} p\right\rfloor+2$. Put $U=\left\{S^{\prime} \subset \mathbb{Z} / p \mathbb{Z} \mid G\left(S^{\prime}\right) \cong G(S)\right\}$. Then we have $\# U=p(p-1)$.

Proof. Let $T$ be a maximal tree of $G(S)$. Let $v_{1}, v_{2}, \ldots, v_{k}$ be the vertices of $T$, and $e_{1}, e_{2}, \ldots, e_{k-1}$ the edges of $T$, where $k=\# S=\left\lfloor\log _{2} p\right\rfloor+2$. Let $A=\left(a_{i j}\right)$ be the $(k-1) \times k$ matrix with $\mathbb{Z}$-entries defined by

$$
a_{i j}=\left\{\begin{aligned}
1 & \left(e_{i} \text { is incident to } v_{j}\right), \\
-2 & \text { (the label of } \left.e_{i} \text { is } v_{j}\right), \\
0 & \text { (otherwise) }
\end{aligned}\right.
$$

Let $A^{\prime}$ be the $(k-1) \times(k-1)$ matrix obtained from $A$ by deleting the $k$ th column. It is known in [1] that

(i) $\operatorname{det}\left(A^{\prime}\right)$ is odd,

(ii) $\left|\operatorname{det}\left(A^{\prime}\right)\right|<2^{k-1}$, and

(iii) $\operatorname{det}\left(A^{\prime}\right)$ is divisible by $p$.

Since $2^{k-2}<p \leq\left|\operatorname{det}\left(A^{\prime}\right)\right|<2^{k-1}$, we have $\left|\operatorname{det}\left(A^{\prime}\right)\right|=p$. This implies that the corank of $A$ with $\mathbb{Z} / p \mathbb{Z}$-entries is exactly equal to 2 .

Let $V=\{\boldsymbol{x} \mid A \boldsymbol{x} \equiv \mathbf{0}(\bmod p)\}$ denote the solution space. By the above argument, we have

$$
V=\left\{\lambda \cdot{ }^{\mathrm{t}}\left(v_{1}, v_{2}, \ldots, v_{k}\right)+\mu \cdot{ }^{\mathrm{t}}(1,1, \ldots, 1) \mid \lambda, \mu \in \mathbb{Z} / p \mathbb{Z}\right\} .
$$

Since the elements of $U$ are identified with the vectors of $V$ whose entries are all distinct. Such a vector is obtained by the condition $\lambda \not \equiv 0(\bmod p)$. Therefore, we have $\# U=p(p-1)$.

Theorem 2.6. Let $S$ and $S^{\prime}$ be subsets of $\mathbb{Z} / p \mathbb{Z}$. Suppose that $G(S)$ and $G\left(S^{\prime}\right)$ are connected with $\# S=\# S^{\prime}=\left\lfloor\log _{2} p\right\rfloor+2$. Then the following are equivalent.

(i) The palette graphs $G(S)$ and $G\left(S^{\prime}\right)$ are isomorphic.

(ii) There exist $\alpha \neq \equiv$ and $\beta \in \mathbb{Z} / p \mathbb{Z}$ such that the affine map $f(x)=\alpha x+\beta$ satisfies $f(S)=S^{\prime}$.

Proof. (ii) $\Rightarrow(\mathrm{i})$. Since $\alpha \not \equiv 0(\bmod p), f: S \rightarrow S^{\prime}$ is a bijection. Furthermore,

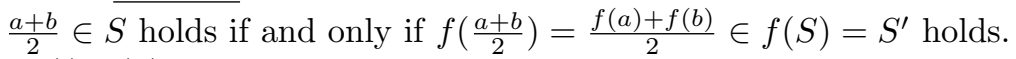

(i) $\Rightarrow$ (ii). By the above argument, we have

$$
U \supset\{f(S) \mid f(x)=\alpha x+\beta, \alpha \not \equiv 0, \beta \in \mathbb{Z} / p \mathbb{Z}\},
$$

where $U$ is the set in Lemma 2.5. Since these two sets have the same number of elements by Lemma 2.5 , they are the same set.

Let $D$ be a diagram of a knot $K$. We regard $D$ as a disjoint union of $\operatorname{arcs}$ whose endpoints are under-crossings. Fox 3 introduced the notion of $p$-colorings: A map $C:\{\operatorname{arcs}$ of $D\} \rightarrow \mathbb{Z} / p \mathbb{Z}$ is a $p$-coloring if $a+b \equiv 2 c(\bmod p)$ holds at every crossing, where $a$ and $b$ are the elements assigned to the under-arcs by $C$, and $c$ is the one to the over-arc. The triple $\{a|c| b\}$ is called the color of the crossing. The assigned element of an arc of $D$ is called the color of the arc. If the color of an $\operatorname{arc}$ is $a$, then the arc is called an $a$-arc.

In a $p$-colored diagram $(D, C)$, the crossing of color $\{a|a| a\}$ is called trivial, and otherwise non-trivial. If $C$ is a constant map, it is called a trivial p-coloring, and otherwise, non-trivial. In other words, a $p$-coloring $C$ is non-trivial if and only if $\# \operatorname{Im}(C)>1$. If a knot $K$ admits a non-trivially $p$-colored diagram $(D, C), K$ is called $p$-colorable. 
For a $p$-colorable knot $K$, we denote by $\mathrm{C}_{p}(K)$ the minimum number of $\# \operatorname{Im}(C)$ for all non-trivially $p$-colored diagram $(D, C)$ of $K$ [5]. For the study of this number, it is helpful to use the palette graph $G(\operatorname{Im}(C))$ of the image $\operatorname{Im}(C) \subset \mathbb{Z} / p \mathbb{Z}$ in the following sense.

Lemma 2.7. If $\{a|c| b\}$ is a non-trivial color of a crossing of a p-colored diagram $(D, C)$, then the palette graph $G(\operatorname{Im}(C))$ has an edge $\{a|c| b\}$.

Proof. Since $a+b \equiv 2 c(\bmod p)$ holds, the lemma follows by definition.

Lemma 2.8. The palette graph $G(\operatorname{Im}(C))$ of a p-colored diagram $(D, C)$ of a knot is connected.

Proof. Let $a$ and $b$ be vertices of $G(\operatorname{Im}(C))$. By definition, we have an $a$-arc and a $b$-arc of $D$. Since $D$ is a diagram of a knot (not a link), we can walk along $D$ from the $a$-arc to the $b$-arc. Let $\left\{a_{i}\left|c_{i}\right| a_{i+1}\right\} \quad(1 \leq i \leq k-1)$ be the colors of nontrivial under-crossings on the path such that $a_{1}=a$ and $a_{k}=b$. Then the vertices $a$ and $b$ in the palette graph are connected by a sequence of edges $\left\{a_{i}\left|c_{i}\right| a_{i+1}\right\}$ $(1 \leq i \leq k-1)$.

Theorem 2.9 ([1] $)$. Any non-trivial p-colored diagram $(D, C)$ of a knot satisfies $\# \operatorname{Im}(C) \geq\left\lfloor\log _{2} p\right\rfloor+2$. Therefore, we have $\mathrm{C}_{p}(K) \geq\left\lfloor\log _{2} p\right\rfloor+2$ for any $p$-colorable knot $K$.

Proof. This follows from Theorem 2.4 and Lemma 2.8

Lemma 2.10. Let $(D, C)$ be a non-trivially $p$-colored diagram of a knot $K$, and $f: \mathbb{Z} / p \mathbb{Z} \rightarrow \mathbb{Z} / p \mathbb{Z}$ an affine map defined by $f(x)=\alpha x+\beta$ with $\alpha \neq \equiv$ and $\beta \in \mathbb{Z} / p \mathbb{Z}$. Then there is a non-trivially $p$-colored diagram $\left(D, C^{\prime}\right)$ of $K$ such that $\operatorname{Im}\left(C^{\prime}\right)=f(\operatorname{Im}(C))$.

Proof. It is easy to see that the composition $C^{\prime}=f \circ C$ is also a non-trivial $p$ coloring of $D$.

Now, we consider the case $p=11$. By Theorem 2.4, if the palette graph $G(S)$ of a subset $S \subset \mathbb{Z} / 11 \mathbb{Z}$ is connected with $\# S>1$, then $\# S \geq 5$.

Theorem 2.11 (4, Theorem 12]). Let $S$ be a subset of $\mathbb{Z} / 11 \mathbb{Z}$. If the palette graph $G(S)$ is connected with $\# S=5$, then $G(S)$ is isomorphic to $G(\{1,4,6,7,8\})$ or $G(\{0,4,6,7,8\})$ as shown in Figure 1 .

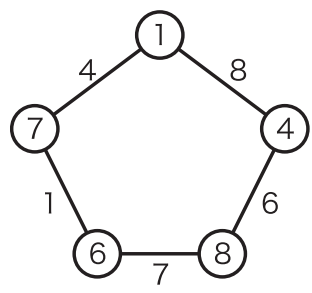

$\mathrm{G}(\{1,4,6,7,8\})$

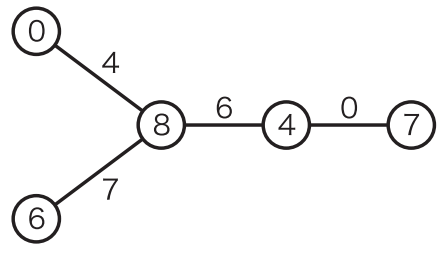

$\mathrm{G}(\{0,4,6,7,8\})$

Figure 1.

By Theorem 2.9 or [10, Theorem 2.4], we have $\mathrm{C}_{11}(K) \geq 5$. The following theorem implies that $\mathrm{C}_{11}(K)=5$ or 6 . 
Theorem 2.12 ([2]). For any 11-colorable knot $K$, there is a non-trivially 11colored diagram $(D, C)$ of $K$ with $\operatorname{Im}(C) \subset\{0,1,4,6,7,8\}$.

Figure 2 shows the palette graph $G(\{0,1,4,6,7,8\})$. By Lemma 2.3 the two graphs in Theorem 2.11 are obtained from this graph by deleting the vertex $a$ and the edges labeled $a$ for $a=0,1$, respectively.

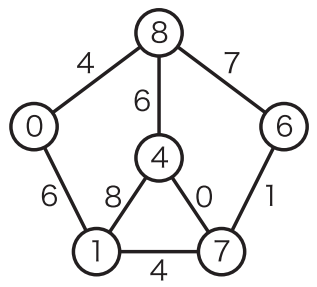

$\mathrm{G}(\{0,1,4,6,7,8\})$

FiguRE 2.

It is useful for our argument to modify Theorem 2.12 slightly as follows.

Lemma 2.13. For any 11-colorable knot $K$, there is an 11-colored diagram $(D, C)$ of $K$ with $\operatorname{Im}(C)=\{0,1,4,6,7,8\}$.

Proof. We may assume that $(D, C)$ satisfies Theorem 2.12 that is, it is a nontrivially 11-colored diagram with $\operatorname{Im}(C) \subset\{0,1,4,6,7,8\}$. We remark that $\# \operatorname{Im}(C) \geq$ 5 by Theorem 2.9

$4,6,7 \in \operatorname{Im}(C)$. Assume that $4 \notin \operatorname{Im}(C)$. It follows that $\operatorname{Im}(C)=\{0,1,6,7,8\}$. The palette graph $G(\operatorname{Im}(C))$ is as shown in the left of Figure 3 by Lemma 2.3 , which contradicts to the connectivity in Lemma 2.8. We can also prove $6,7 \in \operatorname{Im}(C)$ by a similar argument. See the center and right of the figure.
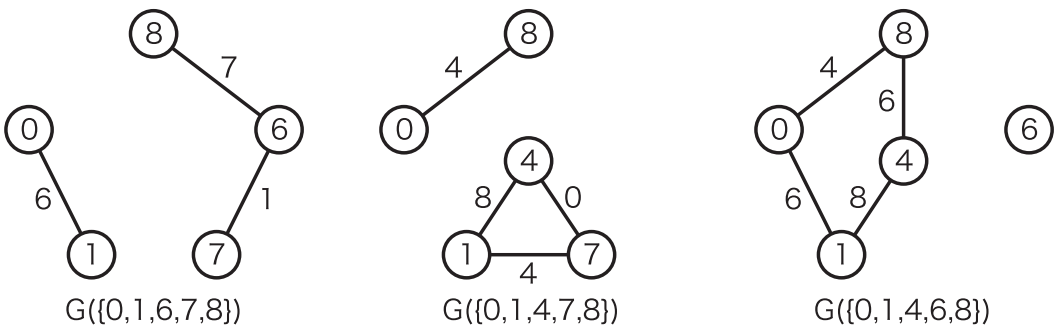

Figure 3.

$0 \in \operatorname{Im}(C)$. Assume that $0 \notin \operatorname{Im}(C)$. It follows that $\operatorname{Im}(C)=\{1,4,6,7,8\}$ and its palette graph is as shown in the left of Figure 1. Then we see that $(D, C)$ has a crossing of color $\{6|1| 7\}$ or $\{1|8| 4\}$. In fact, if we delete the corresponding edges both, the resulting graph becomes disconnected. By deforming the diagram near these crossings as shown in Figure 4, we can produce a 0 -arc. We replace the original diagram with the new one as $(D, C)$.

$1 \in \operatorname{Im}(C)$. Assume that $1 \notin \operatorname{Im}(C)$. Then we have $\operatorname{Im}(C)=\{0,4,6,7,8\}$ and its palette graph is as shown the right of Figure 1. Since $(D, C)$ must have a crossing 


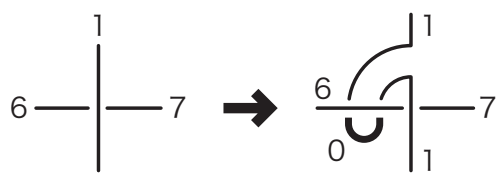

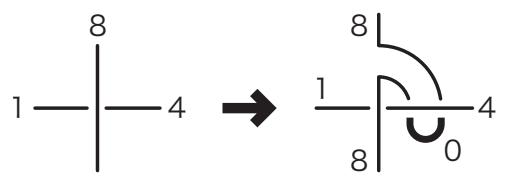

FiguRE 4.

of color $\{0|4| 8\}$ by a similar reason to the above case, we deform the diagram near the crossing to make a 1-arc. See Figure 5.

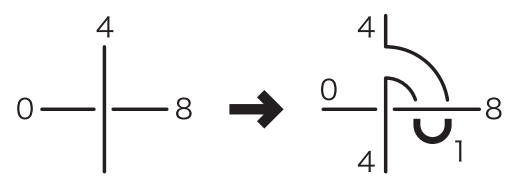

FIGURE 5 .

$8 \in \operatorname{Im}(C)$. Assume that $8 \notin \operatorname{Im}(C)$. Then we have $\operatorname{Im}(C)=\{0,1,4,6,7\}$ and its palette graph is as shown in the left of Figure 6. We remark that the map $f: \mathbb{Z} / 11 \mathbb{Z} \rightarrow \mathbb{Z} / 11 \mathbb{Z}$ defined by $f(x)=7 x+6$ induces the isomorphism between $G(\{0,4,6,7,8\})$ and $G(\{0,1,4,6,7\})$. The existence of such a map is guaranteed by Theorem 2.6. Since $(D, C)$ has a crossing of color $\{4|0| 7\}$, we deform the diagram near the crossing as shown in the right of the figure so that we obtain an 8-arc.
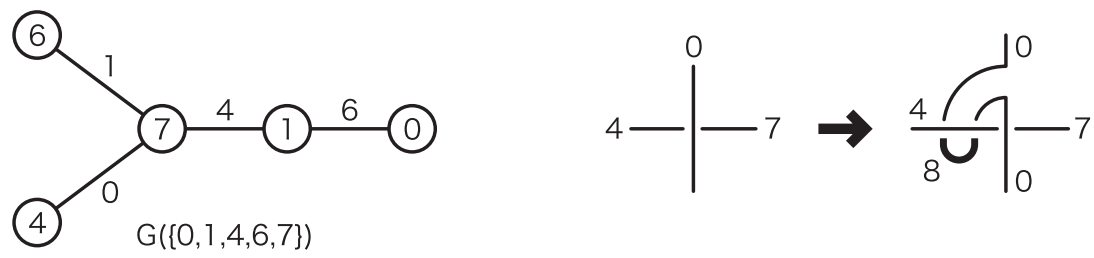

FiguRe 6.

\section{Proof of Theorem 1.1(i)}

Lemma 3.1. For any 11-colorable knot $K$, there is an 11-colored diagram $(D, C)$ of $K$ such that

(i) $\operatorname{Im}(C)=\{0,1,4,6,7,8\}$, and

(ii) there is no crossing of color $\{*|0| *\}$.

Proof. We may assume that $(D, C)$ satisfies Lemma 2.13. There are two types of crossings of $(D, C)$ whose over-arc is a 0 -arc; that is, $\{0|0| 0\}$ and $\{4|0| 7\}$. In fact, in the palette graph $G(\{0,1,4,6,7,8\})$, the only edge labeled 0 connects 4 and 7 .

First, we assume that $(D, C)$ has crossings of color $\{4|0| 7\}$. By deforming the diagram near the crossings as shown in Figure 7 , we can eliminate all the crossings 


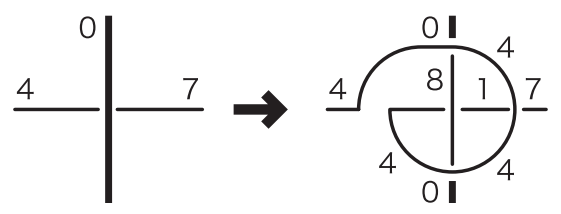

Figure 7.

of color $\{4|0| 7\}$. We remark that the set of colors which are appeared in the diagram does not change.

Next, we assume that $(D, C)$ has a crossing of color $\{0|0| 0\}$, say $x$. Walking along the diagram from $x$, let $y$ be the non-trivial crossing which we meet first. If there are crossings of color $\{0|0| 0\}$ between $x$ and $y$, we replace the original $x$ with the nearest one to $y$. Therefore, we may assume that there is no crossing between $x$ and $y$.

There are two cases with respect to the color of $y$. In fact, in the palette graph $G(\{0,1,4,6,7,8\})$, there are two edges incident to the vertex 0 , which implies that the color of $y$ is $\{0|6| 1\}$ or $\{0|4| 8\}$. In each case, we deform the diagram $(D, C)$ near $x$ and $y$ as shown in Figure 8 , so that the number of crossings of $\{0|0| 0\}$ is decreased. By repeating this process, we obtain a diagram with no crossing of $\{0|0| 0\}$ finally.
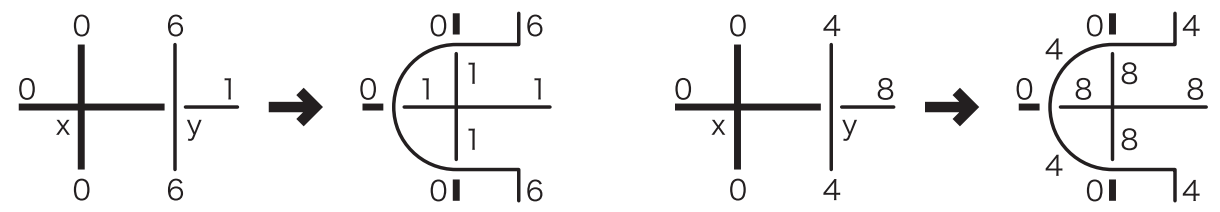

FiguRE 8.

Proof of Theorem 1.1(i). We may assume that $(D, C)$ satisfies Lemma 3.1. If there is a 0 -arc, it is not an over-arc of any crossing, and its endpoints are the undercrossings of color $\{0|4| 8\}$ or $\{0|6| 1\}$. In fact, there are two edges incident to the vertex 0 in $G(\{1,4,6,7,8\})$. We have three cases with respect to the colors of the crossings of the endpoints of a 0 -arc;

(i) $\{0|4| 8\}$ and $\{0|6| 1\}$,

(ii) $\{0|4| 8\}$ both, and

(iii) $\{0|6| 1\}$ both.

For the case (i), we deform the 6 -arc over the crossing of $\{0|4| 8\}$ to eliminate the 0 -arc. See the top of Figure 9 . For the case (ii), we deform one of the crossings of color $\{0|4| 8\}$ as shown in the figure so that we reduce this case to (i). Similarly, for the case (iii), we deform one of the crossings of color $\{0|6| 1\}$ as shown in the figure so that we reduce this case to (i). See the bottom of the figure.

Corollary 3.2. For any 11-colorable knot $K$ and $a \not \equiv b \in \mathbb{Z} / 11 \mathbb{Z}$, there is an 11-colored diagram $(D, C)$ of $K$ with

$$
\operatorname{Im}(C)=\{a, b, 3 a+9 b, 6 a+6 b, 10 a+2 b\} .
$$



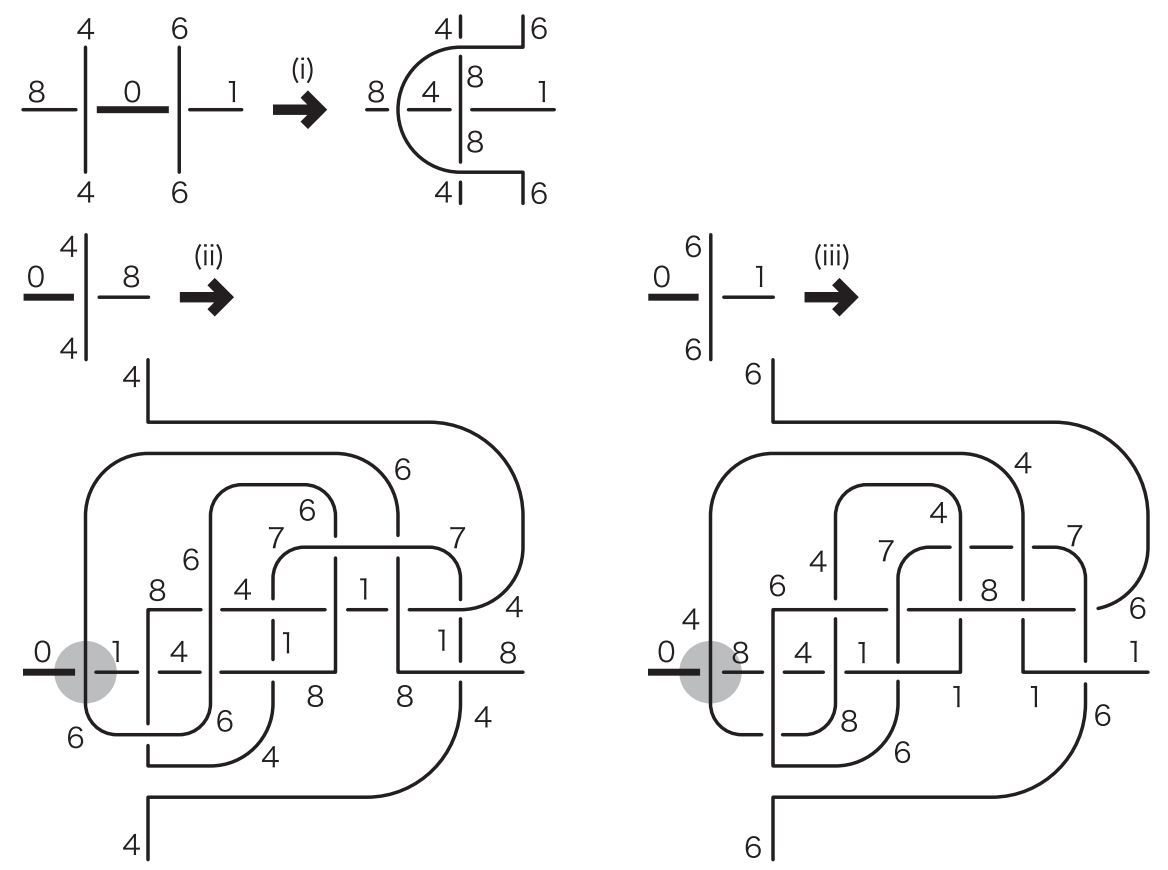

FiguRE 9.

Proof. Let $f: \mathbb{Z} / 11 \mathbb{Z} \rightarrow \mathbb{Z} / 11 \mathbb{Z}$ be the affine map defined by $f(x)=4(b-a)(x-$ 1) $+a$. Since the map $f$ satisfies

$$
f(1)=a, f(4)=b, f(6)=3 a+9 b, f(7)=10 a+2 b, f(8)=6 a+6 b,
$$

we have the conclusion by Lemma 2.10 and Theorem 1.1(i).

\section{Proof of Theorem 1.1(ii)-Part I}

Lemma 4.1. For any 11-colorable knot $K$, there is an 11-colored diagram $(D, C)$ of $K$ such that

(i) $\operatorname{Im}(C)=\{0,1,4,6,7,8\}$, and

(ii) there is no crossing of color $\{6|6| 6\}$.

Proof. We may assume that $(D, C)$ satisfies Lemma 2.13. Assume that $(D, C)$ has a crossing of color $\{6|6| 6\}$, say $x$. Walking along the diagram from $x$, let $y$ be the first non-trivial under-crossing. If there are crossings of color $\{6|6| 6\}$ between $x$ and $y$, then we replace the original $x$ with the nearest one to $y$. Then we have the following:

(i) There is no crossing of $\{6|6| 6\}$ between $x$ and $y$ by assumption.

(ii) Every crossing between $x$ and $y$ is of color $\{0|6| 1\}$ or $\{4|6| 8\}$; for there are exactly two edges labeled 6 in the palette graph $G(\{0,1,4,6,7,8\})$.

(iii) The color of $y$ is $\{6|1| 7\}$ or $\{6|7| 8\}$; for there are exactly two edges incident to the vertex 6 in the palette graph, which are labeled 1 and 7 , respectively.

Assume that there are crossings between $x$ and $y$. Let $z$ be the nearest crossing to $x$ among them. We deform the diagram near $x$ and $z$ as shown in the upper row 
of Figure 10, so that the number of crossings between $x$ and $y$ is decreased. By repeating this process, we may assume that there is no crossing between $x$ and $y$. Then we deform the diagram near $x$ and $y$ as shown in the lower row of the figure to eliminate the color $\{6|6| 6\}$. By repeating this process, we obtain a diagram with no crossing of $\{6|6| 6\}$ finally.
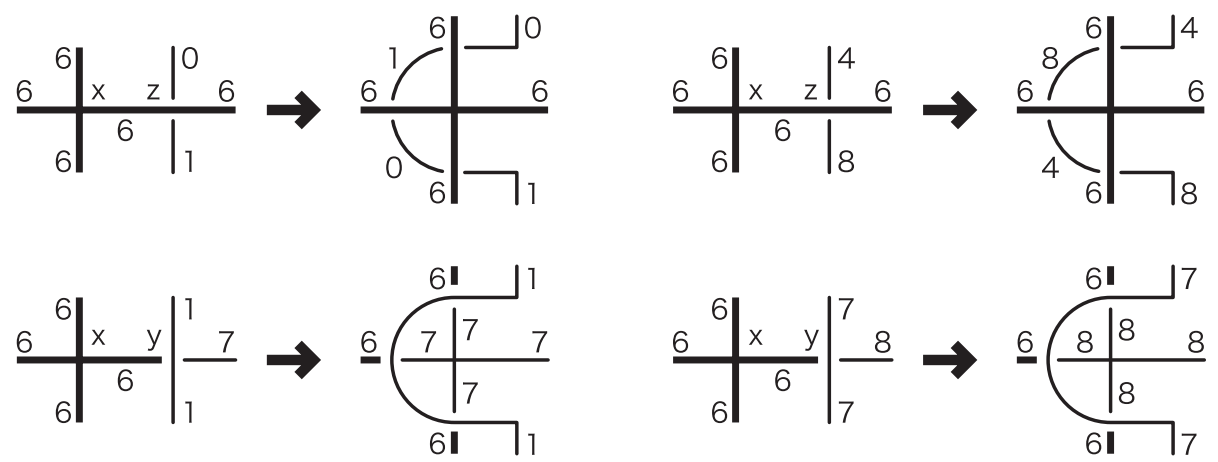

Figure 10.

Lemma 4.2. For any 11-colorable knot $K$, there is an 11-colored diagram $(D, C)$ of $K$ such that

(i) $\operatorname{Im}(C)=\{0,1,4,6,7,8\}$, and

(ii) there is no crossing of color $\{1|1| 1\}$ or $\{6|6| 6\}$.

Proof. We may assume that $(D, C)$ satisfies Lemma 4.1. Assume that $(D, C)$ has a crossing of color $\{1|1| 1\}$, say $x$. Walking along the diagram from $x$, let $y$ be the first non-trivial crossing. If there are crossing of $\{1|1| 1\}$ between $x$ and $y$, then we replace the original $x$ with the nearest one to $y$.

In the palette graph $G(\{0,1,4,6,7,8\})$, there are exactly three edges incident to the vertex 1 whose labels are 4,6 , and 8 , and there is only one edge whose label is 1. Therefore, the color of the crossing $y$ is $\{1|4| 7\},\{1|6| 0\},\{1|8| 4\}$, or $\{6|1| 7\}$.

We deform the diagram near $x$ and $y$ as shown in Figure 11 so that the number of crossings of color $\{1|1| 1\}$ is decreased. By repeating this process, we obtain a diagram with no crossing of $\{1|1| 1\}$.

Lemma 4.3. For any 11-colorable knot $K$, there is a non-trivially 11-colored dia$\operatorname{gram}(D, C)$ of $K$ such that

(i) $\operatorname{Im}(C)=\{0,1,4,6,7,8\}$, and

(ii) there is no crossing of color $\{*|1| *\}$ or $\{6|6| 6\}$.

Proof. We may assume that $(D, C)$ satisfies Lemma 4.2 Assume that $(D, C)$ has a crossing of color $\{*|1| *\}$. Since there is only one edge labeled 1 in the palette graph $G(\{0,1,4,6,7,8\})$, the color of the corresponding crossing is $\{6|1| 7\}$.

There is a 4 -arc in $(D, C)$. We will pull the 4 -arc toward each crossing of $\{6|1| 7\}$. In the process, we can assume that the 4 -arc crosses over several arcs whose colors are $0,1,4,7,8$ missing 6 . In fact, since there is no crossing of $\{6|6| 6\}$, the set of 6 -arcs is a disjoint union of intervals in the plane, and the complement in the plane 

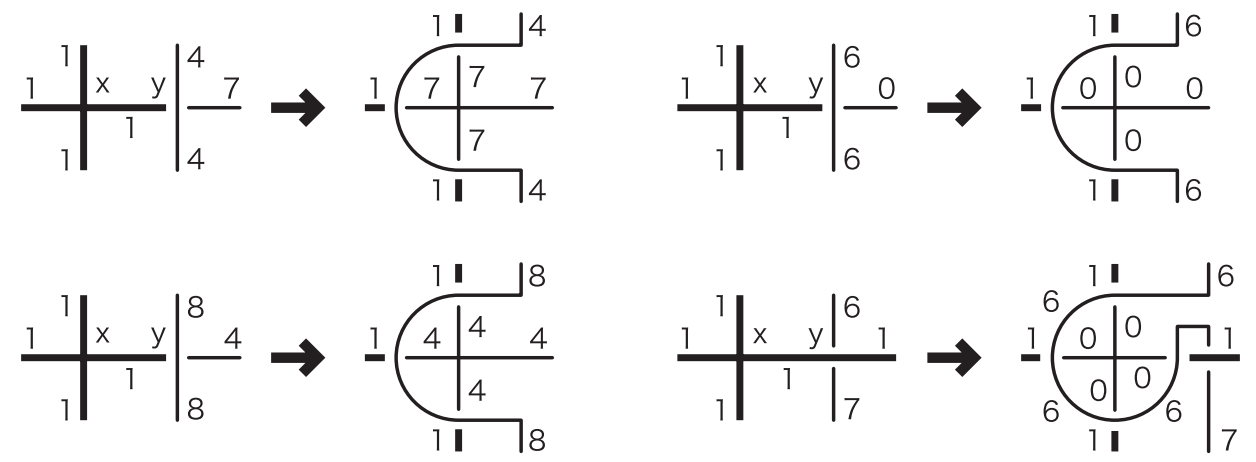

FiguRE 11.

is connected. When the 4 -arc crosses over an $a$-arc for $a=0,1,4,7,8$, we have a pair of new crossings of color

$$
\{a|4| 8-a\}=\{0|4| 8\},\{1|4| 7\},\{4|4| 4\},\{7|4| 1\},\{8|4| 0\},
$$

respectively. See the left of Figure 12 . We remark that any vertex of the palette graph $G(\{0,1,4,6,7,8\})$ other than 6 is 4 itself or incident to an edge labeled 4 .
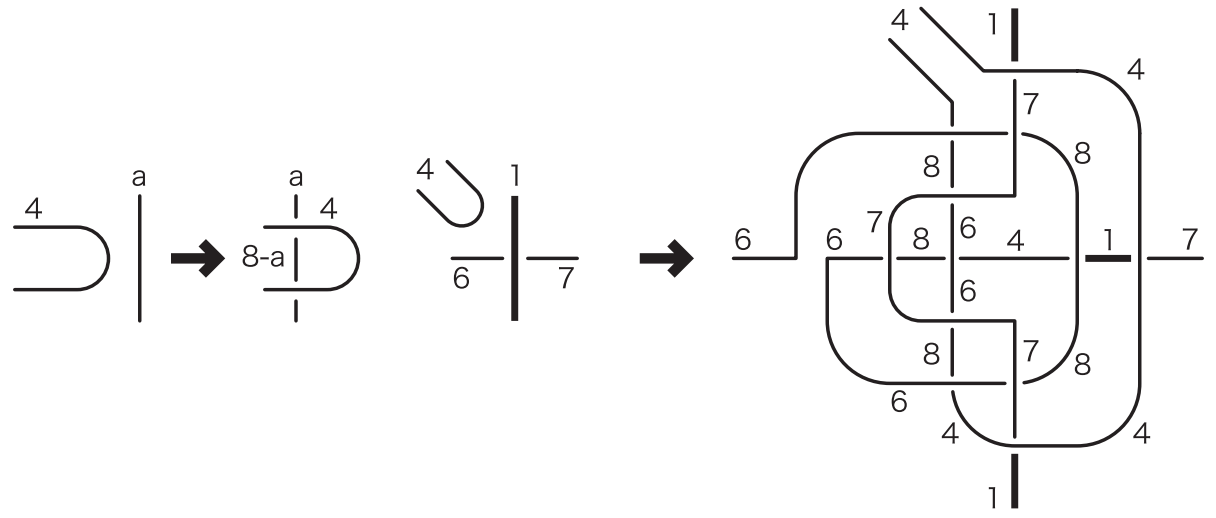

FiguRE 12.

By deforming the diagram near every crossing of $\{6|1| 7\}$ with a 4 -arc as shown in the right of the figure, we obtain a diagram with no crossing of $\{6|1| 7\}$. Then the arcs in the obtained diagram are colored by $0,1,4,6,7,8$ and there is no crossing of $\{*|1| *\}$ or $\{6|6| 6\}$.

Lemma 4.4. For any 11-colorable knot $K$, there is an 11-colored diagram $(D, C)$ of $K$ such that

(i) $\operatorname{Im}(C)=\{0,3,4,6,7,8,10\}$,

(ii) there is no crossing of color $\{6|6| 6\}$, and

(iii) if $\{a|b| c\}$ is the color of a crossing and at least one of $a, b, c$ is 3 or 10 , then it is one of

$$
\{0|3| 6\},\{0|7| 3\},\{3|0| 8\},\{4|7| 10\},\{7|3| 10\},\{3|3| 3\} .
$$


Proof. We may assume that $(D, C)$ satisfies Lemma 4.3. Since there are three edges incident to the vertex 1 in the palette graph $G(\{0,1,4,6,7,8\})$, every crossing with a 1 -arc is of color $\{1|4| 7\},\{1|6| 0\}$, or $\{1|8| 4\}$. If there is a crossing of $\{1|8| 4\}$, we deform the 4-arc near the crossing as shown in Figure 13 to replace the crossing with the one of color $\{1|4| 7\}$. Therefore, we may assume that there is no crossing of $\{1|8| 4\}$.

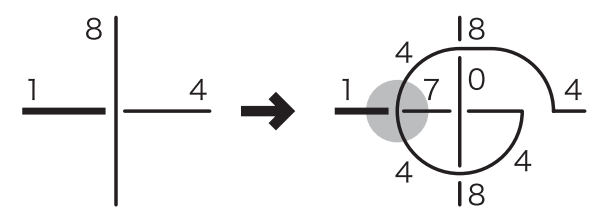

FiguRe 13.

There is a 0 -arc in $(D, C)$. We will pull the 0 -arc toward each crossing of $\{1|4| 7\}$. In the process, we can assume that the 0 -arc crosses over several $a$-arcs for $a \in$ $\{0,1,4,7,8\}$ missing 6 by the same reason in the proof of Lemma 4.3 , that is, there is no crossing of $\{6|6| 6\}$. When the 0 -arc crosses over an $a$-arc, we have a pair of new crossings of color

$$
\{a|0|-a\}=\{0|0| 0\},\{1|0| 10\},\{4|0| 7\},\{7|0| 4\},\{8|0| 3\},
$$

respectively. We remark that the new colors 3 and 10 appear at the crossings of $\{1|0| 10\}$ and $\{3|0| 8\}$. See Figure 14 .
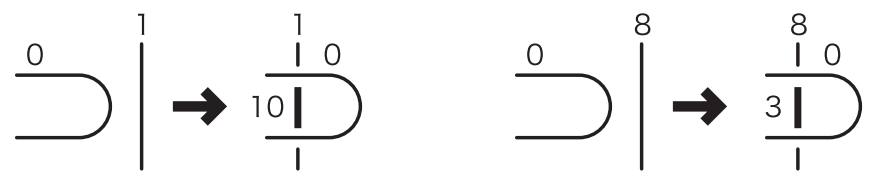

FiguRE 14.

By deforming the diagram near every crossing of $\{1|4| 7\}$ with a 0 -arc as shown in Figure 15. we remove all the crossings of $\{1|4| 7\}$ and produce the color 10 at the crossings of $\{1|0| 10\}$ and $\{4|7| 10\}$.

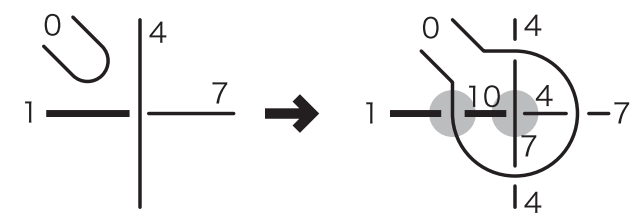

FiguRe 15.

There is a 7 -arc in $(D, C)$. We will pull the 7 -arc toward each 0 -arc. In the process, we can assume that the 7 -arc crosses over several $a$-arcs for $a \in$ 
$\{0,3,4,6,7,8,10\}$ missing 1 ; for there is no crossing of $\{1|1| 1\}$. Then we have a pair of new crossings of

$$
\{a|7| 3-a\}=\{0|7| 3\},\{3|7| 0\},\{4|7| 10\},\{6|7| 8\},\{7|7| 7\},\{8|7| 6\},\{10|7| 4\},
$$

respectively. We remark that the colors 3 and 10 appear at the crossings of $\{0|7| 3\}$ and $\{4|7| 10\}$.

Now, every crossing with a 1 -arc is of color $\{1|6| 0\}$ or $\{1|0| 10\}$. The endpoints of every 1-arc are under-crossings of color

(i) $\{1|6| 0\}$ both,

(ii) $\{1|0| 10\}$ both, or

(iii) $\{1|0| 10\}$ and $\{1|6| 0\}$.

For every 1-arc of type (i), we deform the diagram near the 1-arc equipped with a 7-arc into type (ii) as shown in the left of Figure 16 . Here, the colors 3 and 10 appear at the crossings of $\{0|7| 3\},\{7|3| 10\},\{0|3| 6\},\{3|3| 3\}$, and $\{3|0| 8\}$.
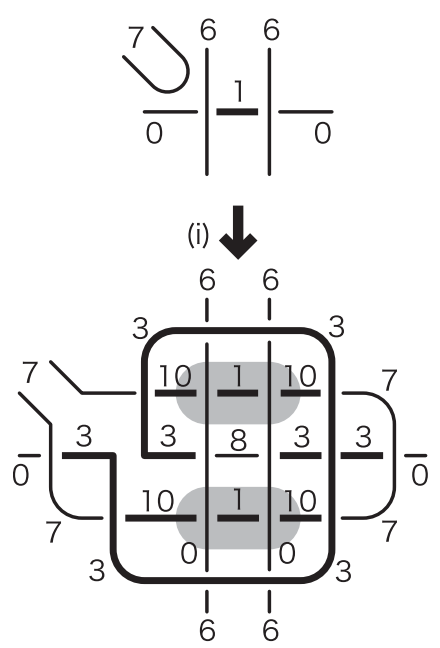

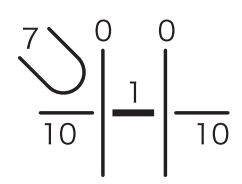

(ii)
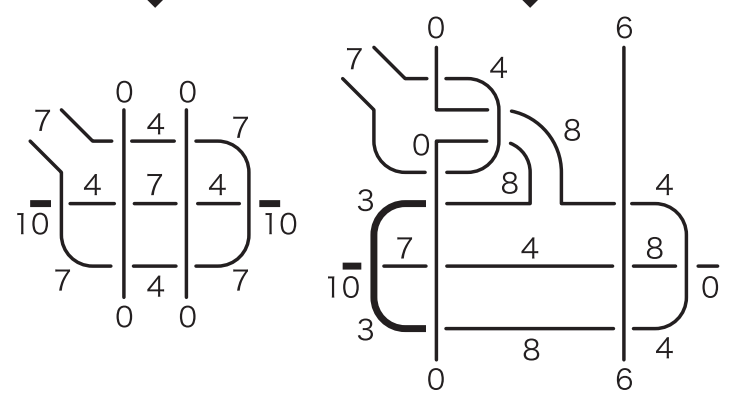

FiguRe 16.

For every 1-arc of type (ii) or (iii), we deform the diagram near the 1-arc with a 7-arc as shown in the center and right of the figure, so that we can remove all the 1-arcs from the diagram. We remark that the colors 3 and 10 appear at the crossings of $\{4|7| 10\}$ for (ii) and $\{3|0| 8\}$ and $\{7|3| 10\}$ for (iii).

Since the original diagram has a 1 -arc, at least one of deformations (i), (ii), and (iii) must happen. Therefore, the obtained diagram has a 10-arc. If the diagram has no 3-arc, the case (ii) must happen. By deforming a neighborhood of a crossing of $\{4|0| 7\}$ similarly to Figures 4 and 5 , we can make a pair of crossings of $\{0|7| 3\}$ so that we have $\operatorname{Im}(C)=\{0,3,4,6,7,8,10\}$.

We remark that the 11-colored diagram $(D, C)$ in Lemma 4.4 has no crossing of color $\{3|10| 6\},\{6|8| 10\}$, or $\{10|10| 10\}$. In particular, there is no crossing whose over-arc is colored 10 . 


\section{Proof of Theorem 1.1(ii)-Part II}

Let $G_{1}$ be the graph obtained from the palette graph $G(\{0,4,6,7,8\})$ by adding two vertices 3 and 10 and five edges

$$
\{0|3| 6\},\{0|7| 3\},\{3|0| 8\},\{4|7| 10\},\{7|3| 10\} .
$$

See Figure 17. In other words, $G_{1}$ is obtained from $G(\{0,3,4,5,6,8,10\})$ by deleting the edges $\{3|10| 6\}$ and $\{6|8| 10\}$.

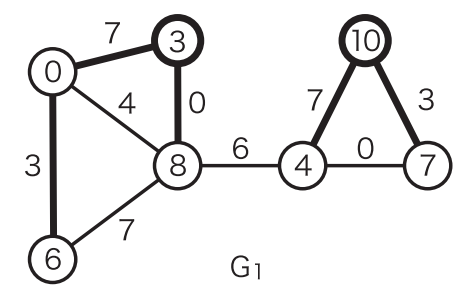

FiguRE 17.

Assume that $(D, C)$ satisfies Lemma 4.4. If $\{a|c| b\}$ is the non-trivial color of a crossing of $(D, C)$, then the palette graph $G_{1}$ has the corresponding edge $\{a|c| b\}$.

Lemma 5.1. For any 11-colorable knot $K$, there is an 11-colored diagram $(D, C)$ of $K$ such that

(i) $\operatorname{Im}(C)=\{0,3,4,6,7,8\}$, and

(ii) there is no crossing of color $\{6|6| 6\}$.

Proof. We may assume that $(D, C)$ satisfies Lemma 4.4 . Since the graph $G_{1}$ has no edge whose label is 10 and $(D, C)$ has no crossing of $\{10|10| 10\}$, we see that there is no crossing of color $\{*|10| *\}$.

Since there are two edges incident to the vertex 10 in $G_{1}$, every crossing with a 10 -arc is of color $\{4|7| 10\}$ or $\{7|3| 10\}$. If there is a crossing of $\{7|3| 10\}$, we deform the 7 -arc near the crossing as shown in the left of Figure 18 to replace the crossing with one of $\{4|7| 10\}$. We remark that the crossings of $\{0|7| 3\}$ and $\{4|0| 7\}$ are also produced. Therefore, we may assume that there is no crossing of $\{7|3| 10\}$.

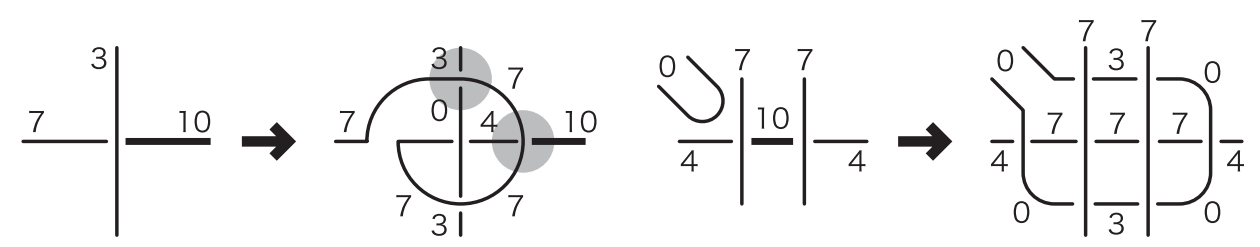

FigURE 18.

There is a 0 -arc in $(D, C)$. We will pull the 0 -arc toward each 10 -arc. In the process, we can assume that the 0 -arc crosses over several arcs whose colors are $0,3,4,7,8$ missing 6 and 10. In fact, since there is no crossing of color

$$
\{3|10| 6\},\{2|6| 10\},\{6|8| 10\},\{6|6| 6\},\{10|10| 10\},
$$


the set of 6 - and 10-arcs is a disjoint union of intervals, and the complement in the plane is connected. When the 0 -arc crosses over an $a$-arc for $a=0,3,4,7,8$, we have a pair of new crossings of color

$$
\{a|0|-a\}=\{0|0| 0\},\{3|0| 8\},\{4|0| 7\},\{7|0| 4\},\{8|0| 3\}
$$

respectively. We remark that any vertex of $G_{1}$ other than 6 and 10 is 0 itself or incident to an edge labeled 0 .

We deform the diagram near every 10 -arc with a 0 -arc as shown in the right of the figure, so that we remove all the 10 -arcs from the diagram. We remark that the crossings of $\{0|7| 3\},\{4|0| 7\}$, and $\{7|7| 7\}$ are produced.

\section{Proof of Theorem 1.1(ii)-Part III}

Lemma 6.1. For any 11-colorable knot $K$, there is an 11-colored diagram $(D, C)$ of $K$ such that

(i) $\operatorname{Im}(C)=\{0,3,4,6,7,8\}$,

(ii) there is no crossing of color $\{3|3| 3\},\{4|4| 4\}$, or $\{6|6| 6\}$.

Proof. We may assume that $(D, C)$ satisfies Lemma 5.1 with $\operatorname{Im}(C)=\{0,3,4,6,7,8\}$. Figure 19 shows the palette graph $G(\{0,3,4,6,7,8\})$, which is obtained from $G_{1}$ by deleting the vertex 10 and its incident edges $\{4|7| 10\}$ and $\{7|3| 10\}$.

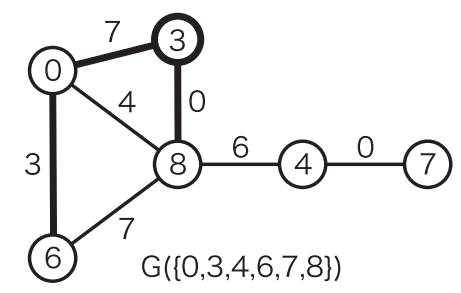

Figure 19.

There is a 0 -arc in $(D, C)$. Similarly to the proof of Lemma 5.1, we can pull the 0 -arc freely without producing new colors. We remark that any vertex of $G(\{0,3,4,6,7,8\})$ other than 6 is 0 itself or incident to an edge labeled 0 . Then we deform the diagram near every 3 - or 4 -arc with a 0 -arc as shown in Figure 20 so that there is no crossing of $\{3|3| 3\}$ or $\{4|4| 4\}$.
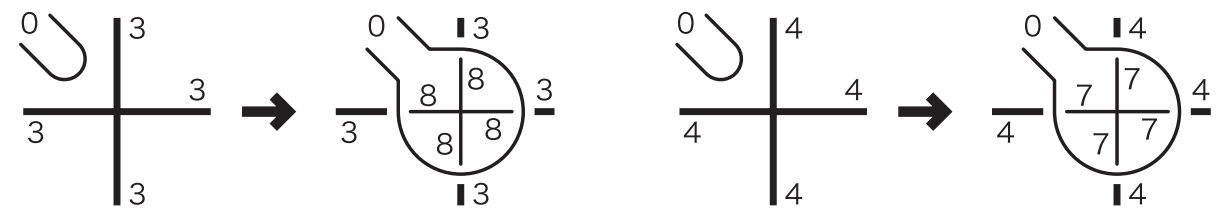

FiguRE 20.

Lemma 6.2. For any 11-colorable knot $K$, there is an 11-colored diagram $(D, C)$ of $K$ such that

(i) $\operatorname{Im}(C)=\{0,3,4,6,7,8\}$, 
(ii) there is no crossing of color $\{*|3| *\},\{4|4| 4\}$, or $\{6|6| 6\}$.

Proof. We may assume that $(D, C)$ satisfies Lemma 6.1. In the palette graph $G(\{0,3,4,6,7,8\})$, there is only one edge whose label is 3 . Therefore, every crossing whose over-arc is 3 has the color $\{0|3| 6\}$.

There is a 7 -arc in $(D, C)$. We will pull the 7 -arc toward each crossing of $\{0|3| 6\}$. Since there is no crossing of $\{4|4| 4\}$, we can assume that the 7 -arc crosses over several arcs whose colors are $0,3,6,7,8$ missing 4 . If the 7 -arc crosses an $a$-arc for $a \in\{0,3,6,7,8\}$, then we have a pair of new crossings of color

$$
\{a|7| 3-a\}=\{0|7| 3\},\{3|7| 0\},\{6|7| 8\},\{7|7| 7\},\{8|7| 6\},
$$

respectively. We remark that any vertex of $G(\{0,3,4,6,7,8\})$ other than 4 is 7 itself or incident to an edge labeled 7 . We deform the diagram near every crossing of $\{0|3| 6\}$ equipped with a 7 -arc as shown in Figure 21 to remove all the crossings of $\{0|3| 6\}$.

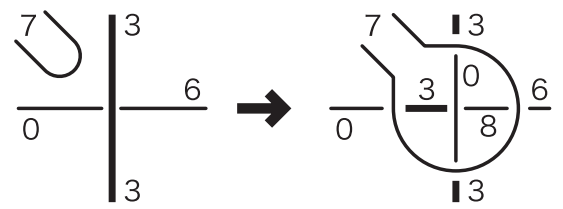

FiguRe 21.

Proof of Theorem 1.1(ii). We may assume that $(D, C)$ satisfies Lemma 6.2. Since there are two edges incident to the vertex 3 in $G(\{0,3,4,6,7,8\})$, every crossing with a 3 -arc is of color $\{3|0| 8\}$ or $\{0|7| 3\}$. Therefore, the endpoints of every 3 -arc are under-crossings of color

(i) $\{3|0| 8\}$ and $\{0|7| 3\}$,

(ii) $\{3|0| 8\}$ both, or

(iii) $\{0|7| 3\}$ both.

For every 3 -arc of type (i), we deform the diagram near the crossing of $\{0|7| 3\}$, which reduces a 3 -arc of type (ii). See the left of Figure 22. Therefore, we may assume that there is no 1-arc of type (i).

To remove a 3-arc of type (ii), We will pull a 7-arc toward the 3-arc. Since there is no crossing of $\{4|4| 4\}$, the 7 -arc can cross over several arcs whose colors are $0,3,6,7,8$ missing 4 similarly to the proof of Lemma 6.2. We remark that when the 7 -arc crosses over an 0 - or 3 -arc, then we have a pair of new crossings of color $\{0|7| 3\}$. We deform the diagram near every 3 -arc of type (ii) with a 7 -arc to remove all the 3 -arcs of type (ii). See the center of the figure.

Now, since every crossing with a 3 -arc is of color $\{0|7| 3\}$, every 3 -arc is of type (iii). We deform the diagram near every 3 -arc of type (iii) with a 7 -arc as shown in the right of the figure so that we obtain a diagram with no 3 -arc.

Corollary 6.3. For any 11-colorable knot $K$ and $a \not \equiv b \in \mathbb{Z} / 11 \mathbb{Z}$, there is an 11-colored diagram $(D, C)$ of $K$ with

$$
\operatorname{Im}(C)=\{a, b, 5 a+7 b, 2 a+10 b, 10 a+2 b\} .
$$



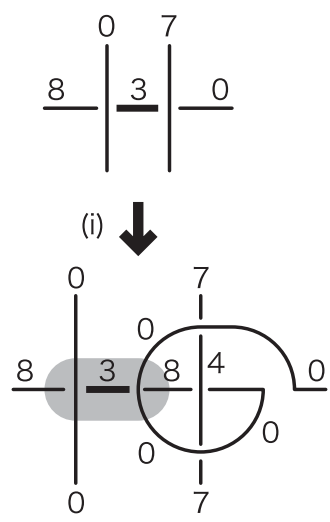
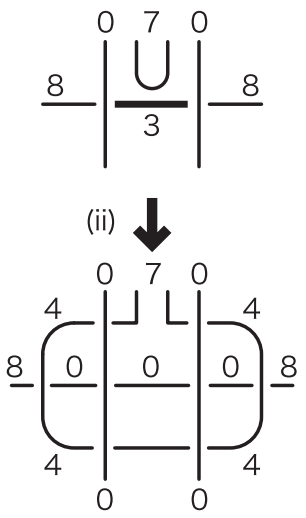
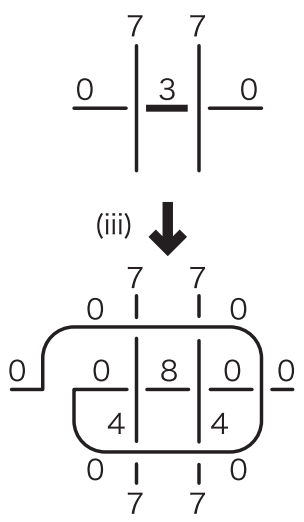

FigURE 22.

Proof. Let $f: \mathbb{Z} / 11 \mathbb{Z} \rightarrow \mathbb{Z} / 11 \mathbb{Z}$ be the affine map defined by $f(x)=3(b-a) x+a$. Since the map $f$ satisfies

$$
f(0)=a, f(4)=b, f(6)=5 a+7 b, f(7)=2 a+10 b, f(8)=10 a+2 b,
$$

we have the conclusion by Lemma 2.10 and Theorem 1.1(ii).

\section{11-COLORABLE RIBBON 2-KNOT}

A ribbon 2-knot 3 is a kind of knotted 2-sphere embedded in $\mathbb{R}^{4}$. Such a 2-knot is presented by a diagram in $\mathbb{R}^{3}$ with only double point circles [18, the $n$-colorability is defined similarly to the classical case by assigning an element of $\mathbb{Z} / n \mathbb{Z}$ to each sheet of the diagram. Refer to 1 for a diagram of a knotted surfaces.

Lemma 7.1. Let $K$ be an 11-colorable ribbon 2 -knot. For each set $S=\{1,4,6,7,8\}$ or $\{0,4,6,7,8\}$, there is an 11-colored diagram of $K$ which satisfies the following.

(i) Every double point circle has a neighborhood as shown in Figure 23, and all the sheets of the diagram other than the small shaded disks are colored by $S$.

(ii) While the color $2 a-b$ of the shaded disk may not belong to $S$, the pair $(a, b)$ must satisfy $2 b-a \in S$.

Proof. Let $A$ be a virtual arc which presents $K$ [16. Since $K$ is 11-colorable, so is $A$. Then there is an 11-colored diagram $(D, C)$ of $A$ with $\operatorname{Im}(C)=S$ by a similar argument in the proof of Theorems 1.1. The diagram of $K$ associated to $(D, C)$ is the desired one [13, 17].

Theorem 7.2. Any 11-colorable ribbon 2-knot satisfies the following.

(i) There is an 11-colored diagram $\left(D_{1}, C_{1}\right)$ of $K$ with $\operatorname{Im}\left(C_{1}\right)=\{1,4,6,7,8\}$.

(ii) There is an 11-colored diagram $\left(D_{2}, C_{2}\right)$ of $K$ with $\operatorname{Im}\left(C_{2}\right)=\{0,4,6,7,8\}$.

Proof. (i) We may assume that $(D, C)$ satisfies Lemma 7.1 for $S=\{1,4,6,7,8\}$. In the left of Figure 23 , the shaded disk is colored $2 b-a$. The pair $(a, b)$ with $a, b, 2 b-a \in S$ and $2 a-b \notin S$ is one of the following:

$$
(a, b)=(4,1),(4,7),(1,7),(1,6),(7,6),(7,8),(6,8),(6,4),(8,4),(8,1) .
$$



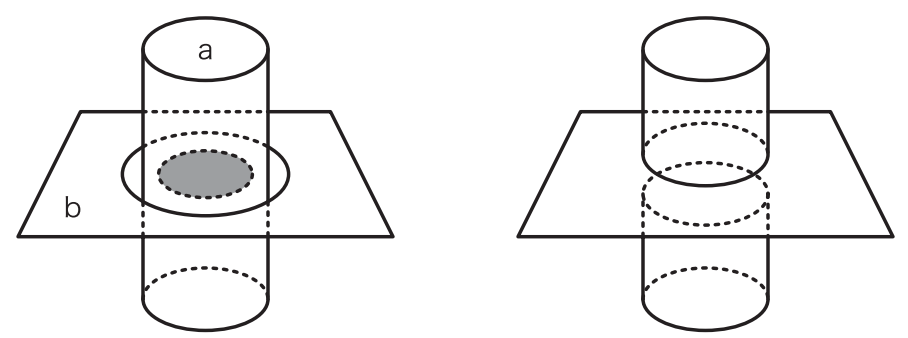

Figure 23.

In fact, each edge $\{x|y| z\}$ in the palette graph $G(S)$ produces such two pairs $(y, x)$ and $(y, z)$.

First, we consider the case $(a, b)=(4,1)$, where the shaded sheet is colored 9 . There is an 8-sheet in $(D, C)$. We pull the 8-sheet toward the 9-sheet without introducing new double points and deform the diagram as shown in the left of Figure 24 to remove the 9 -sheet. We remark that the figure shows a cross-section of the neighborhood of the 9 -sheet. Next, we consider the case $(a, b)=(4,7)$, where the shaded sheet is colored 10 . We deform the horizontal 4 -sheet by surrounding the 10 -sheet, that reduces the case $(a, b)=(4,1)$. See the right of the figure.
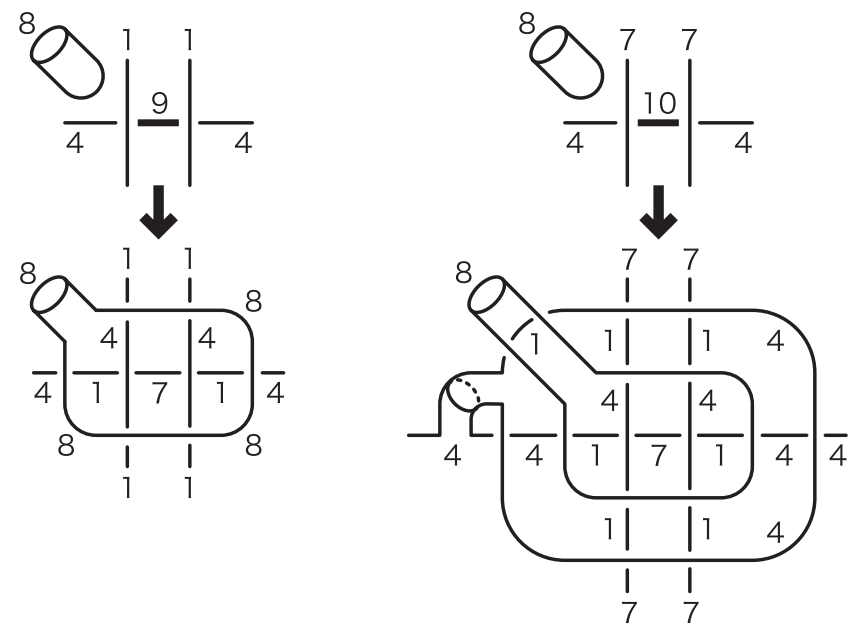

FiguRE 24.

Let $f: \mathbb{Z} / 11 \mathbb{Z} \rightarrow \mathbb{Z} / 11 \mathbb{Z}$ be the affine map defined by $f(x)=9 x+9$. Since we have

$$
f(1)=7, f(4)=1, f(6)=8, f(7)=6 \text {, and } f(8)=4 \text {, }
$$

the cases $(a, b)=(1,7),(7,6),(6,8)$, and $(8,4)$ are obtained from $(a, b)=(4,1)$ by applying $f$ repeatedly, and the cases $(a, b)=(1,6),(7,8),(6,4)$, and $(8,1)$ are obtained from $(a, b)=(4,7)$ similarly.

(ii) We may assume that $(D, C)$ satisfies Lemma 7.1 for $S=\{0,4,6,7,8\}$. The pair $(a, b)$ with $a, b, 2 b-a \in S$ and $2 a-b \notin S$ is one of the following:

$$
(a, b)=(4,8),(7,6),(7,8),(6,8),(6,4), \text { and }(0,7) \text {. }
$$


In fact, each edge $\{x|y| z\}$ in the palette graph $G(S)$ produces such two pairs $(y, x)$ and $(y, z)$ other than $(4,8)$ from $\{0|4| 8\}$ and $(0,4)$ from $\{4|0| 7\}$.

For the case $(a, b)=(4,8)$, we deform the horizontal 4 -sheet by surrounding the shaded 1-sheet as shown in the left of Figure 25 so that we can remove the 1-sheet. The case $(a, b)=(0,7)$ can be similarly proved. See the right of the figure.
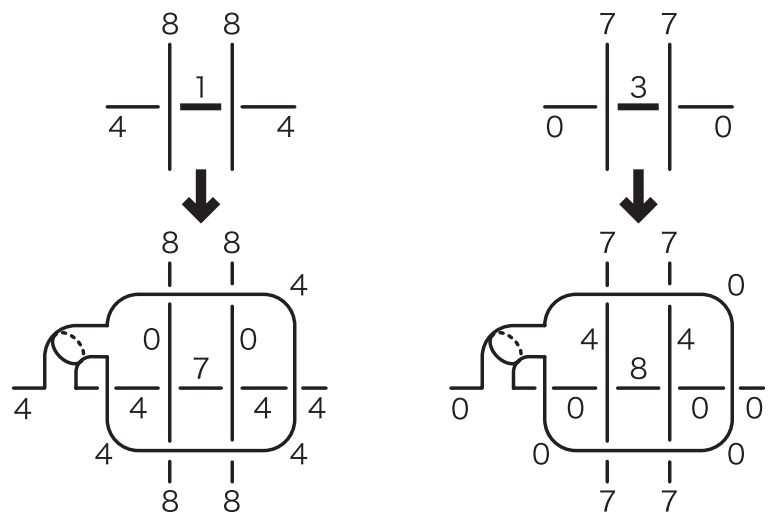

FiguRe 25.

For the case $(a, b)=(7,6)$, we pull a 0 -sheet and deform the diagram as shown in the left of Figure 26. Then we can remove the 5 -sheet without introducing new colors. For the case $(a, b)=(7,8)$, we first deform the horizontal 7 -sheet by surrounding the shaded 9 -sheet, that reduces to the case $(a, b)=(7,6)$.
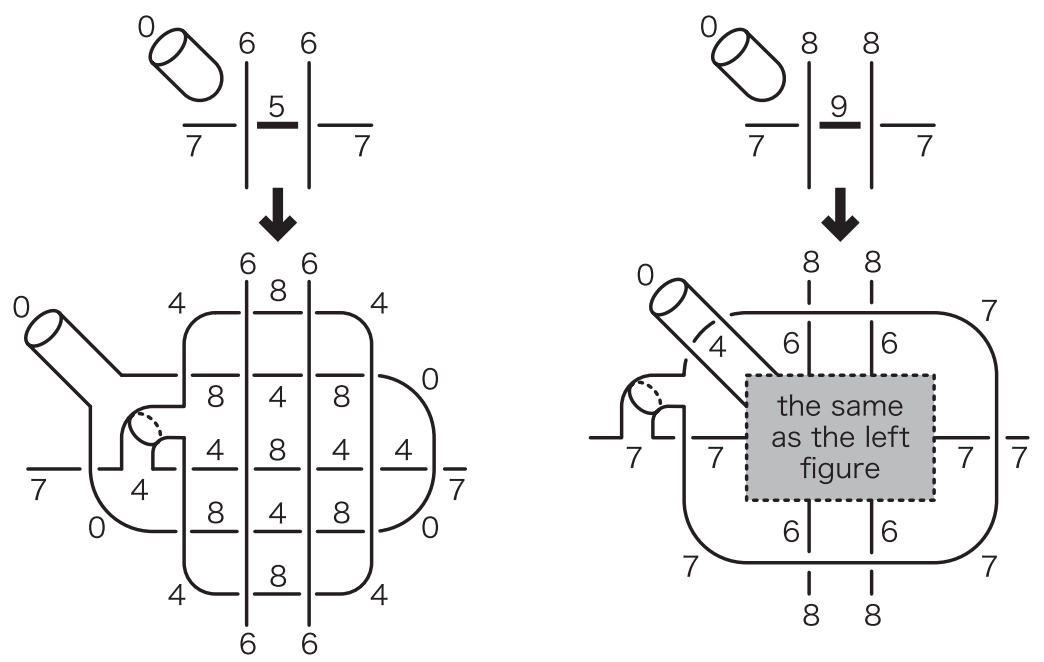

Figure 26.

For the case $(a, b)=(6,8)$, we pull a 7 -sheet and surround the shaded 10-sheet by the 7 -sheet as shown in the left of Figure 27 so that the color 10 is removed. 
For the case $(a, b)=(6,4)$, we pull a 7-sheet toward the shaded 2-sheet and deform the horizontal 6 -sheet to surround the 2 -sheet. Then this case reduces to the case $(a, b)=(6,8)$.
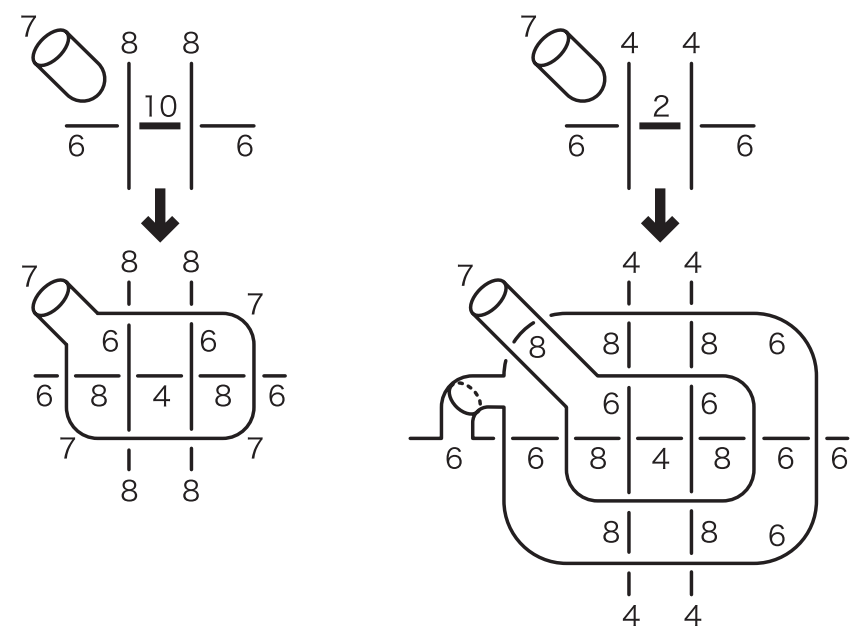

Figure 27.

For a $p$-colorable 2 -knot $K$, we denote by $\mathrm{C}_{p}(K)$ the minimum number of $\# \operatorname{Im}(C)$ for all non-trivially $p$-colored diagrams $(D, C)$ of $K$ [17]. Then the following is an immediate consequence of Theorem 7.2

Corollary 7.3. Any 11-colorable ribbon 2 -knot $K$ satisfies $\mathrm{C}_{11}(K)=5$.

The proof of the following is as same as that of Corollaries 3.2 and 6.3 .

Corollary 7.4. For any 11 -colorable ribbon 2 -knot $K$ and $a \not \equiv b \in \mathbb{Z} / 11 \mathbb{Z}$, there are 11-colored diagrams $\left(D_{1}, C_{1}\right)$ and $\left(D_{2}, C_{2}\right)$ of $K$ with

$$
\begin{aligned}
& \operatorname{Im}\left(C_{1}\right)=\{a, b, 3 a+9 b, 10 a+2 b, 6 a+6 b\}, \text { and } \\
& \operatorname{Im}\left(C_{2}\right)=\{a, b, 5 a+7 b, 2 a+10 b, 10 a+2 b\} .
\end{aligned}
$$

\section{REFERENCES}

[1] J. S. Carter and M. Saito, Knotted surfaces and their diagrams, Mathematical Surveys and Monographs, 55. American Mathematical Society, Providence, RI, 1998.

[2] W. Cheng, X. Jin, and N. Zhao, Any 11-colorable knot can be colored with at most six colors, J. Knot Theory Ramifications 23 (2014), no. 11, 1450062, 25 pp.

[3] R. H. Fox, A quick trip through knot theory, 1962 Topology of 3-manifolds and related topics (Proc. The Univ. of Georgia Institute, 1961) pp. 120-167 Prentice-Hall, Englewood Cliffs, N.J.

[4] J. Ge, X. Jin, L. H. Kauffman, P. Lopes, and L. Zhang, Minimal sufficient sets of colors and minimum number of colors, available at arXiv: 1501.02421

[5] F. Harary and L. H. Kauffman, Knots and graphs. I. Arc graphs and colorings, Adv. in Appl. Math. 22 (1999), no. 3, 312-337.

[6] K. Ichihara and E. Matsudo, A lower bound on minimal number of colors for links, preprint.

[7] L. H. Kauffman and P. Lopes, On the minimum number of colors for knots, Adv. in Appl. Math. 40 (2008), no. 1, 36-53. 
[8] L. H. Kauffman and P. Lopes, The Teneva game, J. Knot Theory Ramifications 21 (2012), no. $14,1250125,17 \mathrm{pp}$.

[9] A. Kawauchi, Lectures on knot theory, Monograph in Japanese, 2007, Kyoritsu Shuppan Co. Ltd.

[10] P. Lopes and J. Matias, Minimum number of Fox colors for small primes, J. Knot Theory Ramifications 21 (2012), no. 3, 1250025, 12 pp.

[11] T. Nakamura, Y. Nakanishi, and S. Satoh, The pallet graph of a Fox coloring, Yokohama Math. J. 59 (2013), 91-97.

[12] T. Nakamura, Y. Nakanishi, and S. Satoh, On effective 9-colorings for knots, J. Knot Theory Ramifications 23 (2014), no. 12, 1450059, 15 pp.

[13] K. Oshiro, Any 7-colorable knot can be colored by four colors, J. Math. Soc. Japan 62 (2010), no. 3, 687-1041.

[14] K. Oshiro and S. Satoh, 7-colored 2-knot diagram with six colors, Hiroshima Math. J. 44 (2014), no. 1, 1-12.

[15] M. Saito, The minimum number of Fox colors and quandle cocycle invariants, J. Knot Theory Ramifications 19 (2010), no. 11, 1449-1456.

[16] S. Satoh, Virtual knot presentation of ribbon torus-knots, J. Knot Theory Ramifications 9 (2000), no. 4, 531-542.

[17] S. Satoh, 5-colored knot diagram with four colors, Osaka J. Math. 46 (2009), no. 4, 909-1173.

[18] T. Yajima, On simply knotted spheres in $\mathbb{R}^{4}$, Osaka J. Math. 1 (1964), 133-152.

Department of Engineering Science, Osaka Electro-Communication University, HatsuCHO 18-8, NEYAGAWA 572-8530, JAPAN

E-mail address: n-takuji@isc.osakac.ac.jp

Department of Mathematics, Kobe University, Rokkodai-Cho 1-1, Nada-ku, Kobe 657 8501, JAPAN

E-mail address: nakanisi@math.kobe-u.ac.jp

Department of Mathematics, Kobe University, Rokkodai-Cho 1-1, Nada-ku, Kobe 657 8501, JAPAN

E-mail address: shin@math.kobe-u.ac.jp 